\title{
ON THE CLASSIFICATION OF SYMMETRIC GRAPHS WITH A PRIME NUMBER OF VERTICES
}

\author{
BY \\ CHONG-YUN CHAO
}

\begin{abstract}
We determine all the symmetric graphs with a prime number of vertices. We also determine the structure of their groups.
\end{abstract}

1. Introduction. A symmetric graph is an undirected graph whose group of automorphisms is transitive on its vertices as well as on its edges. Here, we determine all the symmetric graphs with a prime number $p$ of vertices, i.e., we show that besides the null and complete graphs, for each integer $n$ such that $0<n<p-1$, there exists a symmetric graph with $p$ vertices and degree $n$ if and only if $n$ is even and $n$ divides $p-1$. Also, if the symmetric graphs with $p$ vertices and degree $n$ exist, they all are isomorphic. For each given $p$, we can construct all the symmetric graphs with $p$ vertices. The method of construction which we use here is similar to the one in [2], i.e., we use the properties of a Cayley graph of a cyclic group of order $p$. Our classification depends heavily on a result in [1, Theorem 5, p. 494], i.e., the group of automorphisms of a symmetric graph (nonnull and noncomplete) with $p$ vertices is a Frobenius group. In fact, here we can determine the generators and the defining relations of this Frobenius group. Our classification also confirms a conjecture in [4, p. 144].

2. Definitions and notations. The definitions concerning groups used here are the same as in [3]. Since the definitions concerning graphs are less standard, we state them as follows: The graphs which we consider here are finite, simple, loopless and undirected, i.e., by a graph $X$ we mean a finite set $V(X)$, called the vertices of $X$, together with a set $E(X)$, called the edges of $X$, consisting of unordered pairs $[a, b]$ of distinct elements $a, b \in V(X)$. We also assume that there is at most one edge between two vertices. Two graphs $X$ and $Y$ are said to be isomorphic, denoted by $X \simeq Y$, if there is a one-to-one map $\sigma$ of $V(X)$ onto $V(Y)$ such that $[a \sigma, b \sigma]$ $\in E(Y)$ if and only if $[a, b] \in E(X)$. An isomorphism of $X$ onto itself is said to be an automorphism of $X$. For each given graph $X$ there is a group of all automorphisms, denoted by $G(X)$, where the multiplication is the multiplication of permutations. $X$ is said to be vertex-transitive if $G(X)$ is transitive on $V(X) . X$ is said to be edge-transitive if $G(X)$ is transitive on $E(X) . X$ is said to be symmetric

Presented to the Society, April 9, 1971; received by the editors September 17, 1970.

AMS 1970 subject classifications. Primary 05C25; Secondary 20B25.

Key words and phrases. Classification, symmetric graph, group of automorphisms, generators and defining relations of a group.

Copyright (C) 1971, American Mathematical Society 
if it is both vertex-transitive and edge-transitive. The complete graph (consisting of all possible edges) and the null graph (having $E(X)$ empty) of $n$ vertices have $S_{n}$, the symmetric group of $n$ letters, as their group of automorphisms. Since $S_{n}, n>1$, is doubly transitive, the null graph and the complete graph are symmetric. A symmetric graph is said to be nontrivial if it is neither null nor complete. (When we are only interested in vertex-transitive graphs, it makes no difference whether the graphs are loopless or not.) Let $H$ be an additive abstract finite group and $K$ be a subset of $H$ such that $K$ does not contain the identity of $H$. The Cayley graph of $H$ with respect to $K$ is $X_{H, K}$ with $V\left(X_{H, K}\right)=H$ and $E\left(X_{H, K}\right)=\{[h, h+k] ; h \in H, k \in K\}$. If $K$ is the empty set, then $E\left(X_{H, K}\right)$ is meant to be empty, i.e., $X_{H, K}$ is a null graph. Clearly, the left regular representations of $H$ are contained in $G\left(X_{H, K}\right)$ for any subset $K$ (not containing the identity of $H$ ) in $H$. A graph $X$ is said to be regular if the number of edges incident with each vertex is the same, or $X$ is said to be with degree $m$ if the number of edges incident with each vertex is $m$. The Cayley graphs are regular. A cycle of length $n(>2)$ is a collection of $n$ edges $\left[X_{1}, X_{2}\right],\left[X_{2}, X_{3}\right]$, $\ldots,\left[X_{n}, X_{1}\right]$ where $X_{1}, X_{2}, \ldots, X_{n}$ are distinct. We, sometimes, indicate a cycle of length $n$ by $X_{1}-X_{2}-X_{3}-\cdots-X_{n}-X_{1}$. In [1, p. 493] Theorem 4 states the following:

Let $p$ be a prime, and $G$ be the cyclic group generated by $(123 \ldots p)$. Then Schur's algorithm on $G$ gives all the graphs of $p$ vertices each whose group of automorphisms is transitive.

This theorem implies that if $X$ is a vertex-transitive graph with $p$ vertices, then $X$ is a regular graph with cycles of length $p$ combined together. This is due to the fact that when each basis for the centralizer ring $V(G)$ corresponding to $G$ is a symmetric matrix, it is the adjacency matrix of a cycle of length $p$. (See pp. 492-493 in [1].) Let $D_{p}$ be the dihedral group of order $2 p$ generated by

$$
\begin{aligned}
& R=(012 \ldots(p-1)) \text { and } \\
& D=(0)(1-1)(2-2) \ldots((p-1) / 2-(p-1) / 2)
\end{aligned}
$$

where the negative signs are taken modulo $p$. Then Schur's algorithm on $G$ generated by $R$ and on $D_{p}$ give the same graphs. Hence, we have

Proposition 1. Let $p$ be a prime and $X$ be a vertex-transitive graph with $p$ vertices. Then

(a) $G(X)$ contains the dihedral group $D_{p}$, and

(b) the order of $G(X)$ is even.

We shall repeatedly use Theorem 5 in $[1$, p. 494] which states the following:

Let $X$ be a nontrivial vertex-transitive graph with a prime number $p$ vertices. Then (a) $G(X)$ is solvable; (b) $G(X)$ is a Frobenius group; (c) $G(X)$ is $3 / 2$-fold transitive.

We shall show that if $X$ is a nontrivial symmetric graph with $p$ vertices then this Frobenius group $G(X)$ is metacyclic. 
3. The construction. Our construction here is similar to the one used in [2].

LEMMA 1. Let $p$ be a prime and $n$ be a positive integer such that $n$ is even and $n$ divides $p-1$. Then there exists a symmetric graph with $p$ vertices and degree $n$.

Proof. Let $H=\{0,1,2, \ldots, p-1\}$ be the group of integers modulo $p$, and $A(H)$ be the group of automorphisms of $H$. Then we know that $A(H)$ is a cyclic group of order $p-1$. Say, $A(H)$ is generated by $\sigma$, i.e., $A(H)=\left\{\sigma, \sigma^{2}, \ldots, \sigma^{p-2}, \sigma^{p-1}=e\right\}$. Since $n$ divides $p-1$, we have $p-1=n r$ for some positive integer $r$. Let $\tau=\sigma^{r}$ and

$$
K=\left\{1 \tau, 1 \tau^{2}, \ldots, 1 \tau^{n-1}, 1 \tau^{n}=1\right\}
$$

We claim that if one of the elements in $K$ has its inverse in $K$ (the operation is taken modulo $p$ ), then every element in $K$ has its inverse in $K$. Say, $-\left(1 \tau^{i}\right) \in K$ for some $i, 1 \leqq i \leqq n$. Then, for any $t, 1 \leqq t \leqq n,-\left(1 \tau^{i}\right) \tau^{t-i}=-\left(1 \tau^{t}\right) \in K$ since $K \tau=K$. We claim that $-1 \in K$. Since $n$ is even, $\left(1 \tau^{n / 2}\right) \tau^{n / 2}=1$. If $1 \tau^{n / 2}=j$, then $1=(j) \tau^{n / 2}=j^{2}$. This means $p$ divides $j^{2}-1$. Since $p$ is a prime and $\tau$ is of order $n, j=-1$. It follows that every element in $K$ has its inverse in $K$. We form the Cayley graph, $X_{H, K}$, of $H$ with respect to $K$. Then since the cardinality of $K$ is $n$ and every element in $K$ has its inverse in $K, X_{H, K}$ is a regular graph of degree $n$.

Now we claim that $X_{H, K}$ is a symmetric graph. Since $H$ is abelian and the left regular representation of $H$ is contained in $G\left(X_{H, K}\right)$, the right regular representations (say, generated by $R$ ) belong to $G\left(X_{H, K}\right)$. Consequently, $X_{H, K}$ is vertextransitive. Let $E$ be an arbitrary edge in $X_{H, K}$, then $E=\left[i, i+1 \tau^{j}\right]$ for some $i$ and some $1 \tau^{j} \in K$, and $[0,1] \tau^{j} R^{i}=E$. Since $[0,1] \in E(X)$, it follows that for any two edges in $E\left(X_{H, K}\right)$, there exists an element in $G\left(X_{H, K}\right)$ which takes one to the other, i.e., $X_{H, K}$ is edge-transitive, and it is symmetric.

Let $\langle\tau\rangle$ be the group generated by $\tau=\sigma^{r}$. We know that the order of $\langle\tau\rangle$ is $n$. Two elements, $i$ and $j$ in $H$, are said to be related with respect to $\langle\tau\rangle$ if and only if there is a $\tau^{k} \in\langle\tau\rangle$ such that $i \tau^{k}=j$. Since $\langle\tau\rangle$ is a group, this relation is an equivalence relation. Consequently, $H$ is partitioned into disjoint subsets

$$
\begin{aligned}
& \{0\}, \\
& K=K_{1}=\left\{1 \tau, 1 \tau^{2}, \ldots, 1 \tau^{n-1}, 1 \tau^{n}=1\right\} \\
& K_{2}=\left\{(1 \sigma) \tau,(1 \sigma) \tau^{2}, \ldots,(1 \sigma) \tau^{n}=1 \sigma\right\} \\
& \quad \vdots \quad \vdots \\
& K_{r}=\left\{\left(1 \sigma^{r-1}\right) \tau,\left(1 \sigma^{r-1}\right) \tau^{2}, \ldots,\left(1 \sigma^{r-1}\right) \tau^{n}=1 \sigma^{r-1}\right\}
\end{aligned}
$$

The Cayley graphs $X_{H, K}, X_{H, K_{2}}, \ldots, X_{H, K_{r}}$ are symmetric, and they are pairwise isomorphic since $\sigma^{i-1}$ maps $X_{H, K}$ onto $X_{H, K_{i}}$ isomorphically for $i=2,3, \ldots, r$. Hence, we have

LemMa 2. Let $n, p, H, \sigma$ and $\tau$ be the same as in Lemma 1 , and $K, K_{2}, \ldots, K_{r}$ be (1). Then $X_{H, K}, X_{H, K_{2}}, \ldots, X_{H, K_{r}}$ are symmetric and are pairwise isomorphic. 
LeMmA 3. The Cayley graphs $X_{H, K}, X_{H, K_{2}}, \ldots, X_{H, K_{r}}$ constructed in Lemma 2 are independent of the generators of $A(H)$.

Proof. $A(H)=\left\{\sigma, \sigma^{2}, \ldots, \sigma^{p-1}=e\right\}$ is generated by $\sigma$, i.e., $1 \sigma$ is a primitive root modulo $p$. Let $\mu=\sigma^{i}$ be another generator of $A(H)$, then $i$ and $p-1$ are relatively prime, denoted by $(i, p-1)=1$. Since $p-1=n r$, we have $(i, n)=1$. Let

$$
K_{j}^{\prime}=\left\{\left(1 \sigma^{j}\right) \mu^{r},\left(1 \sigma^{j}\right) \mu^{2 r}, \ldots,\left(1 \sigma^{j}\right) \mu^{n r}=1 \sigma^{j}\right\}
$$

for $j=0,1, \ldots, r-1$. Since $(i, n)=1$, the elements in each of $K_{j}^{\prime}$ are distinct. Also, since $(i, n)=1, K_{j}^{\prime}=K_{j}$ for $j=1,2, \ldots, r$.

\section{The classification.}

LeMma 4. Let $X$ be a symmetric graph with a prime number $p$ of vertices, and $[0, i]$ and $[0, j] \in E(X)$. Then there exists a $\theta \in(G(X))_{0}$ such that $i \theta=j$ where $(G(X))_{0}$ is the subgroup $\{\tau \in G(X) ; 0 \tau=0\}$.

Proof. Since $X$ is edge-transitive, there exists $\sigma \in G(X)$ such that $[0, i] \sigma=[0, j]$. If $0 \sigma=0$ and $i \sigma=j$, then there is nothing to prove. Consider the case $0 \sigma=j$ and $i \sigma=0$. Since $X$ is vertex-transitive, $X$ is a regular graph with cycles of length $p$ combined together. Then $[0, j]$ is on the cycle of length $p$

$$
0-j-2 j-\cdots-(-1) j-0 \text {. }
$$

Let $\theta=\sigma R^{-j} D$. Then clearly, $\theta \in G(X)$,

$$
\begin{aligned}
& 0 \theta=0\left(\sigma R^{-j} D\right)=j\left(R^{-j} D\right)=0, \quad \text { and } \\
& i \theta=i\left(\sigma R^{-j} D\right)=0\left(R^{-j} D\right)=(-j) D=j .
\end{aligned}
$$

LEMMA 5. Let $X$ be a nontrivial symmetric graph with a prime number $p$ of vertices denoted by $H=\{0,1,2, \ldots, p-1\}$, and $H$ be regarded as the group of integers modulo p. If $\sigma \in G(X)$ and $0 \sigma=0$, then $\sigma$ belongs to the group of automorphisms, $A(H)$, of the group $H$, i.e., $(G(X))_{0} \subseteq A(H)$.

Proof. Since $X$ is a vertex-transitive graph with $p$ vertices, $X$ is a regular graph with cycles of length $p$ combined together. There is no loss of generality to assume that $X$ contains the cycle $C_{1}: 0-1-2-\cdots-(p-1)-0$. That is, if $X$ does not contain the cycle $C_{1}$, then we may relabel the vertices so that it contains $C_{1}$ with 0 remaining unchanged. In other words, if $X$ does not contain $C_{1}$, there is an isomorphic map which takes $X$ onto a symmetric graph with $p$ vertices containing $C_{1}$ and 0 is left fixed under the map.

Let $\sigma \in G(X)$ such that $0 \sigma=0$. We want to show $\sigma \in A(H), \sigma \in G(X)$ implies that it is a one-to-one map of the set $H$ onto itself. We only need to show that it is a homomorphism of the group $H$ onto itself, i.e., to show

$$
\sigma=\left(\begin{array}{ccccccc}
0 & 1 & 2 & \cdots & i & \cdots & -1 \\
0 & j & 2 j & \cdots & i j & \cdots & (-1) j
\end{array}\right) .
$$


Suppose not, then we may assume

$$
\begin{gathered}
0 \sigma=0, \quad i \sigma=i j, \quad \text { for } i=1,2, \ldots, k ; 1 \leqq k \leqq p-2, \\
(k+1) \sigma \neq(k+1) j .
\end{gathered}
$$

Say, $(k+1) \sigma=k j+m$ where $m \neq j . X$ contains $C_{1}$ implying $[k, k+1] \in E(X)$. $\sigma \in G(X)$ implies $[k \sigma,(k+1) \sigma]=[k j, k j+m] \in E(X)$. That means $[0, m] \in E(X)$. By Lemma 4, there exists a $\tau \in(G(X))_{0}$ such that $1 \tau=m$. Then $\tau^{-1} R^{k} \sigma R^{-k j}$ $\in(G(X))_{0}$ and $m\left(\tau^{-1} R^{k} \sigma R^{-k j}\right)=m$. If $\tau^{-1} R^{k} \sigma R^{-k j}$ is not the identity $e$, then we have a contradiction since $G(X)$ is a Frobenius group by Theorem 5 in [1]. So, we assume $\tau^{-1} R^{k} \sigma R^{-k j}=e$. Then

$$
(-1) \tau=(-1) R^{k} \sigma R^{-k j}=(k-1) \sigma R^{-k j}=-j .
$$

We claim $(-1) \sigma=-m$. Consider $D \tau D$ where

$$
D=\left(\begin{array}{rrrrrrrrr}
0 & 1 & 2 & \ldots & i & \ldots & -i & \ldots & -1 \\
0 & -1 & -2 & \ldots & -i & \ldots & i & \ldots & 1
\end{array}\right) .
$$

Then we have $0(D \tau D)=0$ and

$$
1(D \tau D)=(-1)(\tau D)=(-j) D=j .
$$

Then either $(D \tau D) \sigma^{-1}$ is $e$, or it contradicts $G(X)$ being a Frobenius group. Hence, we assume $D \tau D=\sigma$. Then

$$
(-1) \sigma=(-1)(D \tau D)=1(\tau D)=m D=-m .
$$

Now we have

Then

$$
\boldsymbol{\sigma}=\left(\begin{array}{cccc}
0 & 1 & \ldots & -1 \\
0 & j & \cdots & -m
\end{array}\right), \quad \text { and } \quad \tau=\left(\begin{array}{cccc}
0 & 1 & \cdots & -1 \\
0 & m & \cdots & -j
\end{array}\right)
$$

and

$$
\begin{aligned}
m\left(\tau^{-1} \sigma R^{m-j}\right) & =1\left(\sigma R^{m-j}\right)=j R^{m-j}=m, \\
(-j)\left(\tau^{-1} \sigma R^{m-j}\right) & =(-1)\left(\sigma R^{m-j}\right)=(-m) R^{m-j}=-j,
\end{aligned}
$$

$$
O\left(\tau^{-1} \sigma R^{m-j}\right)=0 R^{m-j}=m-j .
$$

Since $m \neq j, 0\left(\tau^{-1} \sigma R^{m-j}\right) \neq 0$. Hence, $\tau^{-1} \sigma R^{m-j}$ is not the identity and it leaves $m$ and $-j$ pointwise fixed. That contradicts $G(X)$ being a Frobenius group, and $\sigma \in A(H)$.

THEOREM 1. Let $p$ be a prime and $n$ be an integer such that $0<n<p-1$. Then there exists a nontrivial symmetric graph with $p$ vertices and degree $n$ if and only if $n$ is even and $n$ divides $p-1$.

Proof. If $n$ is even and $n$ divides $p-1$, then, by Lemma 1 , there exists such a graph. Conversely, if a symmetric graph $X$ with $p$ vertices and degree $n$ exists, then $n$ cannot be an odd integer since a vertex-transitive graph is regular and a regular graph with an odd number of vertices cannot have an odd number degree. If 
$p=2$ and $n=1$, then the graph is complete and it is a trivially symmetric graph. We claim that $n$ divides $p-1$. Let $[0, i]$ and $[0, j]$ be any two edges in $E(X)$, then, by Lemma $4, i$ and $j$ belong to the same orbit (set of transitivity), denoted by $U$, of $(G(X))_{0}$. If $[0, k]$ is a non-edge in $X$, then $k \notin U$ since each element in $G(X)$ takes an edge to an edge and a non-edge to a non-edge. Hence, the length of $U$ is $n$. Since by Theorem 5 in [1], $G(X)$ is $3 / 2$-fold transitive, the orbits of $(G(X))_{0}$ have the same length. It follows that $n$ divides $p-1$.

THEOREM 2. Let $p$ be a prime and $n$ be an even integer such that $0<n<p-1$ and $n$ divides $p-1$. Then any two symmetric graphs with $p$ vertices and degree $n$ are isomorphic.

Proof. Let $X$ be a symmetric graph with $p$ vertices and degree $n$. Then $X$ is a regular graph with cycles of length $p$ combined together. We label the vertices of $X$ by $0,1, \ldots, p-1$, and we regard $\{0,1, \ldots, p-1\}=H$ as the group of integers modulo $p$. By Lemma $5,(G(X))_{0}$ is contained in the group of automorphisms, $A(H)$, of $H$. Since $A(H)$ is cyclic, $(G(X))_{0}$ is cyclic. Let $\tau$ be a generator of $(G(X))_{0}$. By Lemma 4, any two edges $[0, i]$ and $[0, j]$ incident with 0 , there exists a $\tau^{k} \in(G(X))_{0}$ such that $i \tau^{k}=j$. This means that the length of the orbit of $(G(X))_{0}$ to which $i$ belongs must be $n$. In fact, the length of every orbit of $(G(X))_{0}$ is $n$ since $G(X)$ is $3 / 2$-fold transitive on $V(X)=H$. Consequently, the order of $(G(X))_{0}=\langle\tau\rangle$ must also be $n$. $[0, i] \in E(X)$ implies $\left[0, i \tau^{k}\right] \in E(X)$ for $k=0,1, \ldots, n-1$. Since $X$ is a regular graph with cycles of length $p$ combined together, $X$ is a Cayley graph $X_{H, K}$ where $K=\left\{i, i \tau, \ldots, i \tau^{n-1}\right\}$. Let $\sigma$ be a generator of $H$, then $i=1 \sigma^{t}$ for some $t$, and $K$ can be written as $\left\{1 \sigma^{t},\left(1 \sigma^{t}\right) \tau, \ldots,\left(1 \sigma^{t}\right) \tau^{n-1}\right\}$.

Let $Y$ be another symmetric graph with $p$ vertices and degree $n$. We also label the vertices of $Y$ by $0,1, \ldots, p-1$, i.e., $V(Y)=H$. Then, by the similar reasons, $(G(Y))_{0}=\langle\theta\rangle$ is a cyclic subgroup of order $n$ in $H$, and $Y$ is a Cayley graph $Y_{H, K}$, where $K^{\prime}=\left\{m, m \theta, \ldots, m \theta^{n-1}\right\}$ and $[0, m] \in E(Y)$. Since $\langle\theta\rangle=H, m=1 \sigma^{s}$ for some $s$, and $K^{\prime}=\left\{1 \sigma^{s},\left(1 \sigma^{s}\right) \theta, \ldots,\left(1 \sigma^{s}\right) \theta^{n-1}\right\}$.

Since $A(H)$ is cyclic, the subgroup of order $n$ in $A(H)$ is unique. Hence, $\langle\tau\rangle=\langle\theta\rangle$, and $K^{\prime}=\left\{1 \sigma^{s},\left(1 \sigma^{s}\right) \tau, \ldots,\left(1 \sigma^{s}\right) \tau^{n-1}\right\}$. By Lemma $2, X \simeq Y$. By Lemma $3, X$ and $Y$ are so constructed that they do not depend on the choice of the generators $\sigma$ of $H$.

In the proof of Theorem 2, we have shown the following:

COROLlARY 1. Let $X$ be a symmetric graph with a prime number $p$ of vertices and degree $n$ where $n$ is even, $0<n<p-1$ and $n$ divides $p-1$. Then $(G(X))_{0}=\langle\tau\rangle$ is $a$ cyclic group of order $n$ generated by $\tau$ which can be regarded as an automorphism of the group of integers modulo $p$.

\section{The group.}

THEOREM 3. Let $X$ be the symmetric graph with a prime number $p$ of vertices and degree $n$ where $0<n<p-1, n$ is even and $n$ divides $p-1$. Then

(1) $G(X)$ is a Frobenius group. Hence $G(X)$ is 3/2-fold transitive. $G(X)$ contains the dihedral group of order $2 p$. 
(2) $|G(X)|=n p$.

(3) $\langle R\rangle$ is the Frobenius kernel of $G(X)$. Hence, $\langle R\rangle$ is normal in $G(X)$ where $R=(012 \ldots(p-1))$.

(4) $G(X)$ is metacyclic.

(5) $G(X)$ is a semidirect product of the cyclic subgroups $\langle R\rangle$ and $(G(X))_{0} . G(X)$ is generated by $R$ and $\sigma$ with defining relations

$$
R^{p}=e, \quad \sigma^{n}=e, \quad \sigma R \sigma^{-1}=R^{r}
$$

where $r^{n} \equiv 1 \bmod p$.

(6) All Sylow subgroups of $G(X)$ are cyclic.

Proof. (1) was proved in [1, Theorem 5]. Our Proposition 1 shows the dihedral group of order $2 p$ belonging to $G(X)$.

(2) Since $G(X)$ is vertex-transitive $|G(X)|$ is equal to the product of $\left|(G(X))_{0}\right|$ and $p$ by Corollary 5.2 .1 on p. 56 in [3].

(3) Let $N$ be the subset of $G(X)$ consisting of the identity together with those elements which fix no vertices. Then we know that, by Frobenius' theorem (see p. 292 in [3]), $N$ is a normal subgroup of $G(X)(N$ is called the Frobenius kernel of $G(X)$ ), and the order of $N$ is equal to the index of $(G(X))_{0}$ in $G(X)$, i.e., $|N|=p$ by (2). Since $N$ clearly contains $\langle R\rangle$ and $|\langle R\rangle|=p, N=\langle R\rangle$.

(4) Since $G(X) /\langle R\rangle \simeq(G(X))_{0}, G(X) /\langle R\rangle$ is abelian. Hence $\langle R\rangle$ contains the commutator subgroup $(G(X))^{2}$ of $G(X) . G(X)$ containing the dihedral group implies $(G(X))^{2} \neq\{e\}$. Since $\langle R\rangle$ is a cyclic group of order $p$, we have $\langle R\rangle=(G(X))^{2}$. Hence, $G(X)$ is metacyclic.

(5) Since $\langle R\rangle$ is normal in $G(X)$ and $\langle R\rangle \cap(G(X))_{0}=\{e\}, G(X)=\langle R\rangle(G(X))_{0}$. Since $(G(X))_{0}$ is a cyclic group of order $n, G(X)$ is generated by $R$ and $\sigma$ where $\sigma$ is a generator of $(G(X))_{0}$, and $\sigma$, by Corollary 1 , belongs to the group of automorphisms of integers modulo $p$. Since $\langle R\rangle$ is normal in $G(X), \sigma R \sigma^{-1}=R^{r}$ for some $r$. Then, using the fact that $\sigma$ belongs to the group of automorphisms of integers modulo $p$, and $\sigma$ is of order $n$, we have

$$
\begin{aligned}
\sigma R \sigma^{-1} & =\left(\begin{array}{lllll}
0 & 1 & \ldots & k^{n-1} & \ldots \\
0 & k & \ldots & 1 & \ldots
\end{array}\right)\left(\begin{array}{lllll}
0 & 1 & \ldots & k & \ldots \\
1 & 2 & \ldots & (k+1) & \ldots
\end{array}\right)\left(\begin{array}{ccccc}
0 & 1 & \ldots & (k+1) & \ldots \\
0 & k^{n-1} & \ldots & k^{n-1}(k+1) & \ldots
\end{array}\right) \\
& =\left(\begin{array}{cccc}
0 & 1 & \ldots \\
k^{n-1} & k^{n-1}(k+1) & \ldots
\end{array}\right)=\left(\begin{array}{cccc}
0 & 1 & \ldots \\
k^{n-1} & k^{n-1}+1 & \ldots
\end{array}\right)
\end{aligned}
$$

where we use the fact $k^{n}=1$, and all the operations are taken modulo $p$. That means $r=k^{n-1}$, and $r^{n}=\left(k^{n-1}\right)^{n}=\left(k^{n}\right)^{n-1}=1$, i.e., $r^{n} \equiv 1 \bmod p$, and we have obtained the defining relations.

(6) It follows from Theorem 9.4.3 on p. 146 in [3].

6. Summary and examples. For any given odd prime $p, p-1$ is even and is a product of primes $p-1=2^{t_{1}} q_{2}^{t_{2}} \cdots q_{j}^{t_{j}}$. From this decomposition we can find all even integers $n_{i}$ such that $2 \leqq n_{i}<p-1$ and $n_{i}$ divides $p-1$. Say, there are $k$ of them; and for each $i=1,2, \ldots, k$, we have $p-1=n_{i} r_{i}$ for some integer $r_{i}$. Let $\sigma$ be a generator of $A(H)$ which is the group of automorphisms of the group $H$ of integers 
modulo $p$, then $\sigma$ is of order $p-1$. Let $\tau_{i}=\sigma^{r_{i}}$, then the order of $\tau_{i}$ is $n_{i}$. Let

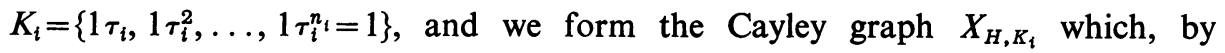
Theorems 1 and 2, is the unique (up to isomorphism) symmetric graph with $p$ vertices and degree $n_{i}$. With the null graph and the complete graph, we have obtained all symmetric graphs with $p$ vertices. With the help of Theorem 3 , we know the structure of each of their groups of automorphisms.

The case of $p=11$. Since $(p-1) / 2$ is a prime, the only symmetric graphs of 11 vertices are null graph, complete graph and cycles of length 11. Their groups of automorphisms are $S_{11}, S_{11}$ and $D_{11}$ respectively.

The case of $p=13$. Besides the null graph and the complete graph of 13 vertices (their group of automorphisms is $S_{13}$ ), the symmetric graphs with 13 vertices are with degree 2,4 and 6 . Let $H=\{0,1,2, \ldots, 12\}$ be the group of integers modulo 13 . The group of automorphisms $A(H)$ of $H$ is of order 12 generated by $\sigma$ where $1 \sigma=2$ ( 2 is a primitive root modulo 13). Hence, we have $\sigma=\left(\begin{array}{lll}1 & 24836121195107\end{array}\right)$ and $A(H)=\left\{\sigma, \sigma^{2}, \ldots, \sigma^{12}=e\right\}$.

Degree 2. Each $X_{H,\{i,-i\}}, i=1,2, \ldots, 6$, is a cycle of length 13 . Clearly, they are pairwise isomorphic. $G\left(X_{H,\{i,-i\}}\right)=D_{13}, i=1,2, \ldots, 6$.

Degree 4. Let $K_{1}=\left\{1 \sigma^{3}=8,1 \sigma^{6}=12,1 \sigma^{9}=5,1 \sigma^{12}=1\right\} . \quad X_{H, K_{1}}$ is shown in Figure 1.

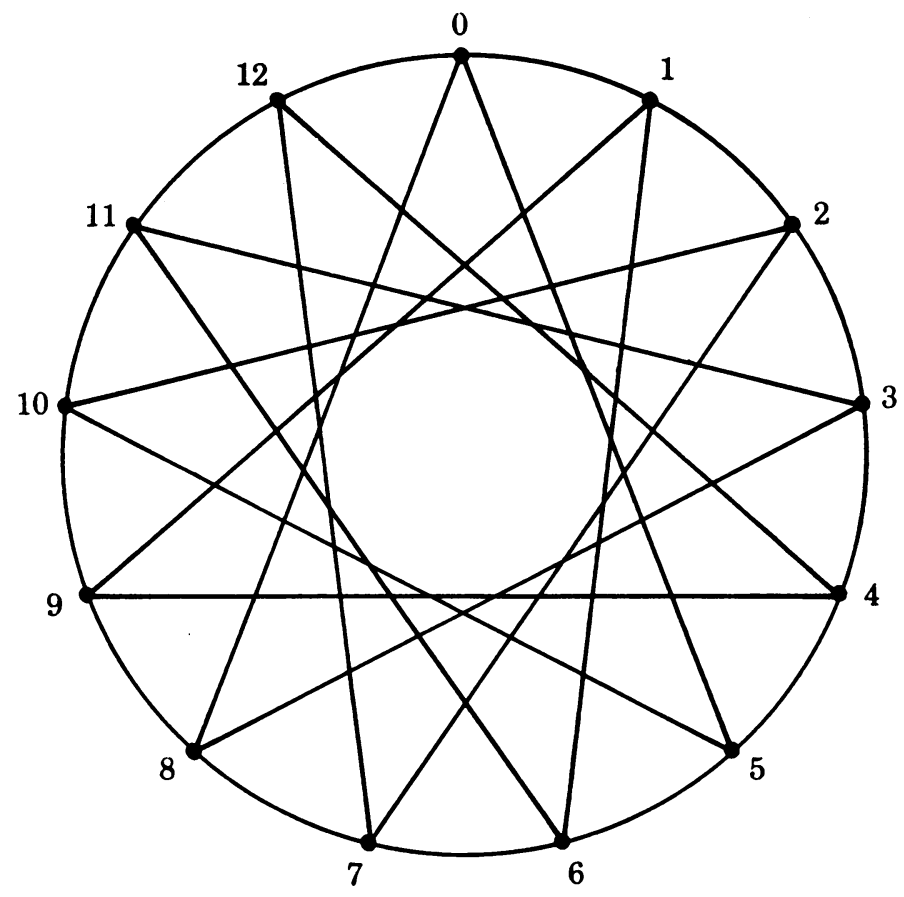

Figure 1 
$K_{2}=\left\{1 \sigma^{4}=3,1 \sigma^{7}=11,1 \sigma^{10}=10,1 \sigma=2\right\}$ and $X_{H, K_{1}} \simeq X_{H, K_{2}}$ where the isomorphic map is $\sigma$. Similarly, $K_{3}=\left\{1 \sigma^{5}=6,1 \sigma^{8}=9,1 \sigma^{11}=7\right.$ and $\left.1 \sigma^{2}=4\right\}$ and $X_{H, K_{1}} \simeq X_{H, K_{3}}$ where the isomorphic map is $\sigma^{2}$.

$G\left(X_{H, K_{i}}\right), i=1,2,3$, is generated by $R$ and $\tau=\sigma^{3}$ where

$$
R=(012 \ldots 12), \text { and } \tau=\left(\begin{array}{llll}
1 & 8 & 12 & 5
\end{array}\right)\left(\begin{array}{llll}
2 & 3 & 11 & 10
\end{array}\right)\left(\begin{array}{lll}
4 & 6 & 9
\end{array}\right)
$$

with $R^{13}=e, \tau^{4}=e$ and $\tau R \tau^{-1}=R^{5}$. The order of $G\left(X_{H, K_{i}}\right)$ is $52, i=1,2,3$.

Degree 6. Let $K_{4}=\left\{1 \sigma^{2}=4,1 \sigma^{4}=3,1 \sigma^{6}=12,1 \sigma^{8}=9,1 \sigma^{10}=10,1 \sigma^{12}=1\right\} . X_{H, K_{4}}$ is shown in Figure 2.

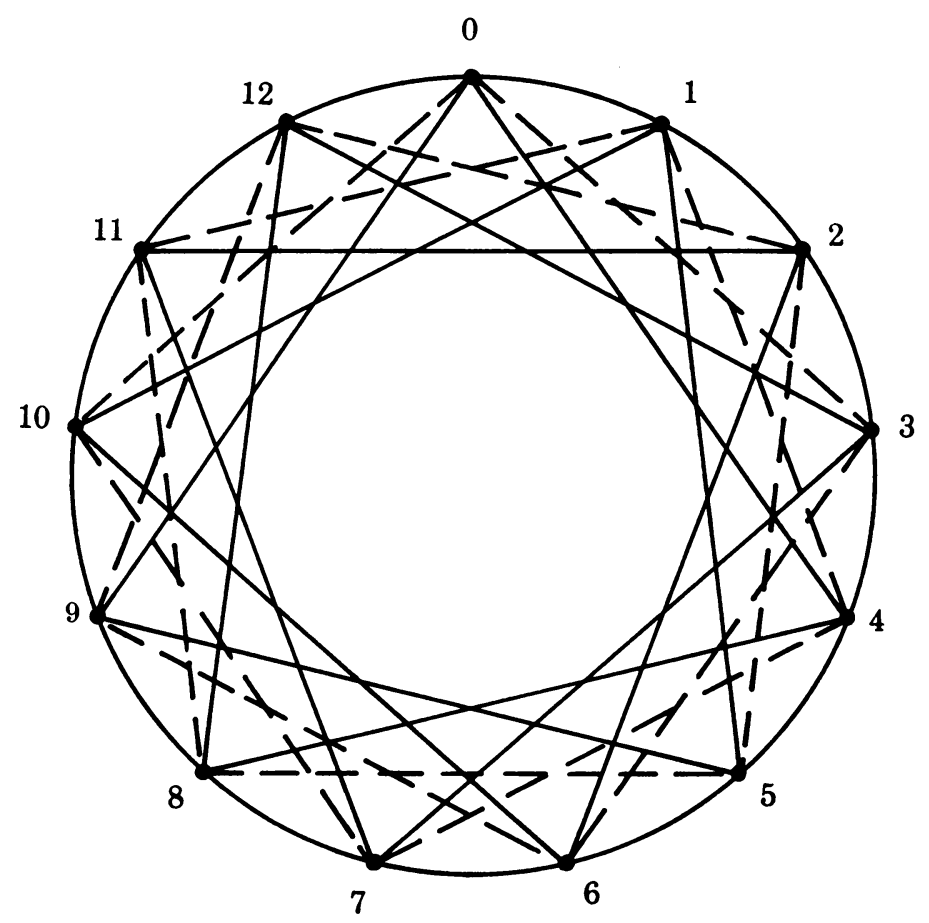

FIGURe 2

$K_{5}=\left\{1 \sigma^{3}=8,1 \sigma^{5}=6,1 \sigma^{7}=11,1 \sigma^{9}=5,1 \sigma^{11}=7,1 \sigma=2\right\}$ and $X_{H, K_{4}} \simeq X_{H, K_{5}}$ where the isomorphic map is $\sigma$.

$G\left(X_{H, K_{j}}\right), j=4,5$, is generated by $R$ and $\theta=\sigma^{2}$ where

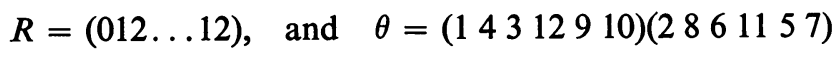

with $R^{13}=e, \theta^{6}=e$ and $\theta R \theta^{-1}=R^{10}$. The order of $G\left(X_{H, K_{4}}\right)$ is 78 . 


\section{REFERENCES}

1. C. Y. Chao, On groups and graphs, Trans. Amer. Math. Soc. 118 (1965), 488-497. MR 31 \#1296.

2. - A note on two set-transitive permutation groups, Proc. Amer. Math. Soc. 17 (1966), 953-955.

3. M. Hall, Jr., The theory of groups, Macmillan, New York, 1959. MR 21 \#1996.

4. J. Turner, Point-symmetric graphs with a prime number of points, J. Combinatorial Theory 3 (1967), 136-145. MR 35 \#2783.

University of Pittsburgh,

Pittsburgh, Pennsylvania 15213 